\title{
Polymeric Nanoencapsulation of Indocyanine green for Photodynamic Therapy Technique
}

\author{
Mohammadreza Saboktakin* \\ Nanomedicine department, NanoBMat Company, Germany \\ *Corresponding author: Mohammadreza Saboktakin, Nanomedicine department, NanoBMat Company, Germany. \\ To Cite This Article: Mohammadreza Saboktakin. Polymeric Nanoencapsulation of Indocyanine green for Photodynamic Therapy Technique. \\ Am J Biomed Sci \& Res. 2019 - 3(1). AJBSR.MS.ID.000630. DOI: 10.34297/AJBSR.2019.03.000630
}

Received: May 03, 2019 | Published: May 16, 2019

\begin{abstract}
Indocyanine green (ICG) has been exploited as a photosensitizer for use in cancerous phototherapy including breast, brain, and skin tumors. Although ICG is of particular advantage for use in cancer phototherapy, it adversely tends to disintegrate in aqueous medium and such degradation can be markedly accelerated by light irradiation (photodegradation) and/or heating (thermal degradation). Furthermore, ICG after administered intravenously will be readily bound with blood proteins and hence leads to only $2 \pm 4 \mathrm{~min}$ of plasmatic half-life. Among various pharmaceutical polymers, poly (lactic-co-glycolic acid) (PLGA), PEG, Chitosan are the best-defined biomaterials with FDA approval for drug encapsulation due to its biocompatibility, biodegradability, and controllability for drug release. This review shows that novel applications of biodegradable polymers as nanocarrier for ICG to improve of physicochemical properties of ICG such as Half-life.
\end{abstract}

Keywords: Biodegradable polymers; Indocyanine green (ICG); Photodynamic therapy (PDT); Cancer treatment

Abbrevations: NIR: Near-Infrared; ICG: Indocyanine Green; PDT: Photodynamic Therapy; ROS: Reactive Oxygen Species; PTT: Photothermal Therapy; FDA: Food and Drug Administration; PEG: Polyethylene Glycol; PLGA: Poly Lactic-Co-Glycolic Acid; FA: Folic Acid; CPNPs: Calcium Phosphosilicate Nanoparticles; RES: Reticuloendothelial System; DOX: Doxorubicin; MDR: Multidrug Resistance; HA: Hyaluronic Acid; nGO: NanoGraphene Oxide; NPs: Nanoparticles; PFP: Perfluoropentane; PT-OCT: Photothermal Optical Coherence Tomography; RB: Rose Bengal; CS: Chitosan; DS: Dextran Sulfate; TPP: Tripolyphosphate; MTZ: Metronidazole; Dox: Doxorubicin; LDH: Layered Double Hydroxide; EPR: Enhanced Permeability and Retention; DLVO: Derjaguin-Landau- Verwey-Overbeek; FDA: Food and Drug Administration

\section{Introduction}

As mentioned earlier, the encapsulation of ICG in the polymeric construct enhances its physicochemical attributes such as half-life, bioavailability, stability and pharmacokinetic characteristics, and reduces the ease of degradation. Moreover, these versatile ICG-encapsulated NIR theranostic nanoparticles also offer numerous other advantages such as significant targeting ability and sensitivity in tumor imaging, which facilitate them in exploring other innovative therapeutic applications. Herein, we elaborate on the discussion of ICG encapsulation and its delivery using various ground-breaking polymer nanocomposites with a set of examples. Among various approaches of breast cancer treatment, near-infrared (NIR)-mediated phototherapy is one of the most promising strategies for serving as a supplement to traditional cancer therapies since it can provide

i. Enhanced tissue penetration efficacy as compared with that operated by visible light

ii. Moderate toxicity to normal cells/tissues through use of targeted photosensitive agents and/or spatially controlled light irradiation [1].
Generally speaking, phototherapy is carried out by hyperthermia and/or reactive oxygen species (ROS) generated from the photosensitizers under light illumination in the presence of oxygen that the former may cause thermal ablation of cancer cells (i.e., photothermal therapy; PTT), while the latter may seriously interfere cellular metabolism and thus trigger programed cell death (i.e., photodynamic therapy; PDT) [2,3]. No matter which mechanism is utilized, the photosensitizer plays a key role in the effectiveness of phototherapy. Indocyanine green (ICG) is an U.S. Food and Drug Administration (FDA)-approved tricarbocyanine dye which enables to absorb and fluoresce in the region of $650 \pm 850 \mathrm{~nm}$. Currently, in addition to serving as a fluorophoric agent for use in diagnostic purposes such as NIR image-guided oncologic surgery [4], fluorescence angiography [5], and lymph node detection of cancer [6], ICG has been exploited as a photosensitizer for use in cancerous phototherapy including breast, brain, and skin tumors [7-9] since it enables to produce heat and ROS (i.e., singlet oxygen) upon NIR irradiation. Although ICG is of particular advantage for use in cancer phototherapy, it adversely tends to disintegrate in aqueous medium and such 
degradation can be markedly accelerated by light irradiation (photodegradation) and/or heating (thermal degradation) [10].

Furthermore, ICG after administered intravenously will be readily bound with blood proteins and hence leads to only $2 \pm 4 \mathrm{~min}$ of plasmatic half-life $[11,12]$. These circumstances seriously hinder the applicability of ICG in the clinic and thus a strategy that enables to enhance the aqueous stability and target efficiency of ICG is certainly needed for ICG-mediated therapy. Nanomedicine may offer a feasible means for usage of ICG without aforementioned defects since it may provide merits of enhanced bioavailability, improved stability, and security for the payload [13]. In terms of the materials used for making drug carrier, polymer is often considered as the preferred candidate since it can be manipulated to tailor the properties and/or functionalities required by the product [14]. Among various pharmaceutical polymers, poly (lactic-co-glycolic acid) (PLGA) is the copolymer of poly(lactic acid) and poly(glycolic acid) and is one of the best defined biomaterials with FDA approval for drug encapsulation due to its biocompatibility, biodegradability, and controllability for drug release [15]. Polyethylene glycol (PEG), another FDA-approved polymer with characteristics of nontoxicity and less immunogenicity, is frequently used for surface modification of drug carrier since the retention time of the PEG-coated particle in the blood circulation can be markedly increased [16].

Taken all together, we aim to develop an anti-HER2 ICG-encapsulated PEG-coated PLGA nanoparticles (HIPPNPs) for targeted phototherapy of HER2-expressing breast cancer cells. The use of ICG by implantation of HIPPNPs instead of naked molecules is advantageous because the polymeric carrier (i.e., HIPPNP) may

a. Potentially protect the entrapped ICG from degradation caused by external stimuli such as light, heat, and/or extreme $\mathrm{pH}$ [17]

b. Preciously localize the therapeutic region to reduce off-target toxicity

c. Provide accurate estimation for the efficacy of ICG-mediated phototherapy.

There are many of reports that ICG encapsulators, such as (poly (lactic-co-glycolic acid) (PLGA) nanoparticles (diameter $\sim 360 \mathrm{~nm}$ ) and silica-polymer composite microcapsules (diameter $\sim 0.6$ to $2 \mu \mathrm{m}$ ) improve the molecular instability of ICG and prolong its plasma half-life $[18,19]$. However, both of these nanoparticles are limited in size for in vivo tumor imaging depending on their EPR effects. Recently, several publications have reported promising results using smaller nanoparticles to encapsulate ICG for in vivo imaging. For example, Zheng et al. [20] developed ICG encapsulated PLGA-lipid nanoparticles conjugated with folic acid (FA) and demonsstrated their use as NIR contrast agents for tumor diagnosis and targeted imaging. Altinoglu et al. [21] also synthesized biodegradable calcium phosphosilicate nanoparticles (CPNPs) and demonstrated that small size $(16 \mathrm{~nm})$ ICG-encapsulating CPNPs have significantly better contrast agent optical properties than free fluorophores for tumor imaging [21]. Other inorganic delivery systems using silica nanoparticles have been developed to encapsulate ICG, and the
ICG-SiO2 nanoparticles have the potential to be used as contrast agents for optical NIR imaging as well [22].

Among these nanocarriers, micelles are one of the successful types of drug delivery systems for in vivo applications due to their small size (approximately 10-100 nm), which reduce clearance by the reticuloendothelial system (RES) and allow for an enhanced EPR effect $[23,24]$. Therefore, the encapsulation and stabilization of ICG dye as a contrast agent in micellar systems is of particular interest. For example, Pluronic F-127 (PF-127) polymeric micelles are approved by the FDA and have been successfully demonstrated to encapsulate and stabilize ICG as an NIR contrast agent for optical imaging $[25,26]$. Encapsulation of ICG within various micellar systems was also investigated by Kirchherr and co-workers, and they found many micellar systems improved the optical properties and stability of the ICG [27]. More interestingly, Zheng et al. [28] have recently reported a dual-functional ICG-PL-PEG agent with several unique features for optical imaging and photo-therapy [28]. This may emerge as a new strategy for combining tumor treatment and diagnosis together, using nanovectors with ICG. In summary, this editorial discussed recent developments in nanocarrier ICG contrast agents for NIR optical imaging.

Here just some of the areas are collected in terms of subjects and interests but it is hoped that every reader will find something of interest to them. Manchanda et al. [29] have recently reported the fabrication of a novel polymer nanoparticle delivery system with simultaneously entrapped indocyanine green (ICG) and doxorubicin (DOX). This system has potential applications for combined chemotherapy and hyperthermia. Research in our group showed that simultaneous use of ICG and DOX with localized hyperthermia can produce the same effect as that achieved by larger doses of chemotherapy alone. In this study, we explored the potential of dual-agent PLGA nanoparticles (ICG-DOXPLGANPs) to overcome multidrug resistance (MDR) mechanisms in cancer cells by increasing intracellular drug concentrations via nanoparticle uptake. ICG-DOX-PLGANPs were prepared by the $\mathrm{O} / \mathrm{W}$ emulsion solvent evaporation method. The dominant processing parameters that control particle size and drug entrapment efficiencies of ICG and DOX were PLGA concentration, PVA concentration and initial drug content. We optimized our previous formulation based on those parameters.

Entrapment efficiency of the optimized ICG-DOX-PLGANPs was measured by fluorescence measurements using the DMSO burst release procedure. The internalization of ICG-DOX- PLGANPs by three cancer cell lines was visualized by confocal laser microscopy and fluorescence microscopy. Cytotoxicity was assessed using the SRB assay. The nanoparticles produced by optimal formulation had sizes of $135 \pm 2 \mathrm{~nm}$, ( $\mathrm{n}=3$ ) with a low poly-dispersity index $(0.149 \pm 0.014, n=3)$ and a zeta potential of $-11.67 \pm 1.8 \mathrm{mV}$. Drug loading was approximately $3 \% \mathrm{w} / \mathrm{w}$ for ICG and $4 \% \mathrm{w} / \mathrm{w}$ for DOX $(n=3)$. Cellular uptake of ICG and DOX from ICG-DOX-PLGANPs in DOX-resistant MESSA/Dx5 cancer cells was higher compared to free ICG and free DOX treatment. However, the same phenomenon was not observed in MES-SA and SKOV-3 cancer cell lines. The SRB cytotoxicity results show that ICG-DOX-PLGANPs are more toxic than 
free DOX in DOX-resistant cell lines. In the development of drugs for intra-articular administration, sustained-release formulations are desirable because it is difficult to maintain the effect of conventional injections due to immediate drug leakage from the joint cavity.

In this study, a sustained-release poly (lactic-co-glycolic acid) (PLGA) microsphere formulation for intra-articular administration containing indocyanine green (ICG) as a model drug was prepared to follow its fate after intra-articular administration in rats with a real-time in-vivo imaging system. ICG administered as an aqueous solution leaked from the joint cavity in a short time and was excreted outside the body within 1-3 d. However, ICG in the sustained-release formulation was retained in the joint cavity and released for 2 weeks. Next, a sustained-release formulation containing PLGA microspheres in a hyaluronic acid (HA) gel formulation was prepared [30] (Figure 1).

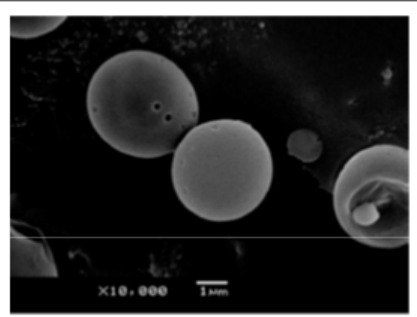

Figure 1: Observation of PGLA microspheres by SEM.

Ronak et al. [31] have been developed and characterized multifunctional biodegradable and biocompatible poly lactic-co-glycolic acid (PLGA) nanoparticles loaded with indocyanine green (ICG) as an optical-imaging contrast agent for cancer imaging and as a photothermal therapy agent for cancer treatment. PLGA-ICG nanoparticles (PIN) were synthesized with a particle diameter of $246 \pm 11 \mathrm{~nm}$, a polydispersity index of $0.10 \pm 0.03$, and ICG loading efficiency of $48.75 \pm 5.48 \%$. PIN were optically characterized with peak excitation and emission at 765 and $810 \pm 5 \mathrm{~nm}$, a fluorescence lifetime of $0.30 \pm 0.01 \mathrm{~ns}$, and peak absorbance at $780 \mathrm{~nm}$. The cytocompatibility study of PIN showed $85 \%$ cell viability till $1-\mathrm{mg} / \mathrm{ml}$ concentration of PIN. Successful cellular uptake of ligand conjugated PIN by prostate cancer cells (PC3) was also obtained. Both phantom-based and in vitro cell culture results demonstrated that PIN

a) Have the great potential to induce local hyperthermia (i.e., temperature increase of 8 to $10^{\circ} \mathrm{C}$ ) in tissue within $5 \mathrm{~mm}$ both in radius and in depth;

b) Result in improved optical stability, excellent biocompatibility with healthy cells, and a great targeting capability;

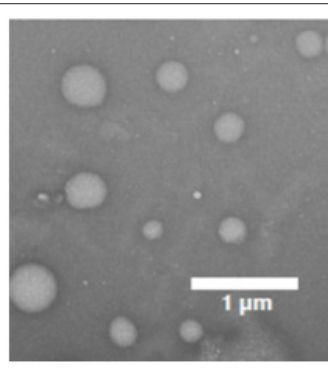

Figure 2: TEM image of presenting of morphology of PIN. c) Have the ability to serve as an image contrast agent for deep-tissue imaging in diffuse optical tomography (Figure 2).

Nowadays, a new technique such as photodynamic therapy (PDT) is used to achieve effective root canal disinfection and eliminate Enterococcus faecalis as the most prevalent species associated with secondary endodontic infections and treatment failures. Employment of an optimized nontoxic photosensitizer (PS) such as indocyanine green (ICG) is a crucial part of this technique; the current study aimed at improving ICG photodynamic properties through conjugation of ICG into nano-graphene oxide (nGO) as a new PS, to evaluate the antimicrobial effects of nGO/ICG against $\mathrm{E}$. faecalis. The PDT based on ICG, at the concentration loaded in nGO, showed a significant reduction in the number of E. faecalis at a lower concentration of ICG. In conclusion, the application of nGO as a new drug delivery system, in addition to the anti-bacterial property, offers other benefits such as cost beneficial outcomes due to using the lower dye concentration (less toxicity), and less tooth discoloration. The nGO/ICG-PDT could be proposed as a new approach to treat endodontic infections, alone or in combination with conventional root canal treatments [32] (Figure 3).

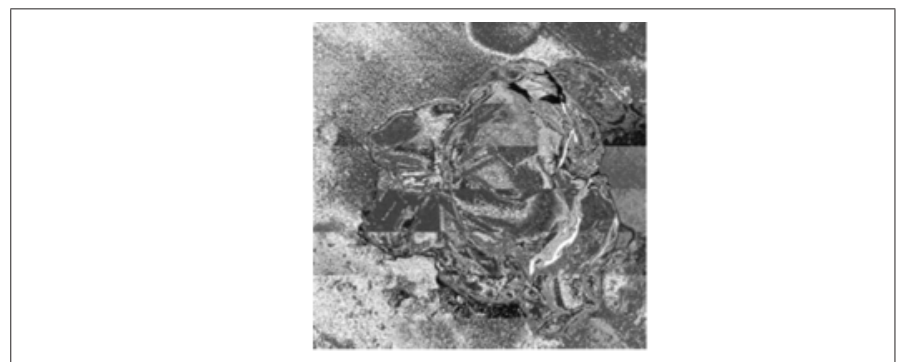

Figure 3: The SEM image of the nGO.

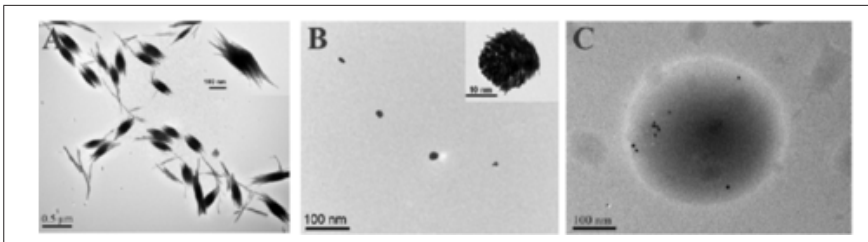

Figure 4 : The TEM images of (A) Nanorod-Bundled WO, (B) Granulated.

Zheng et al. [33] had successfully constructed the fluorescence image guided photothermal therapy reagents based on (WO+ICG) @PLGA) nanoparticles. In our design, to improve their tumor targeting, the macrophages as cell-based biocarriers were employed for delivery the (WO+ICG) @PLGA nanoparticles. The macrophages carried these nanoparticles still had phagocytosis to tumor cells and could also secret plenty of anti-tumor cytokines for immunotherapy of carcinoma. They also further elucidated the superior solid tumor suppression efficiency of the (WO+ICG) @PLGA nanoparticles loaded macrophages targeting biocarriers delivery system in vivo. The system achieved a significant antitumor effect by activating immunotherapy and photothermal therapy in vivo. Hence, such kind of (WO+ICG) @PLGA nanoparticles loaded macrophages delivery system has great potential applications as targeting biocarriers loading drugs and imaging agents for visual-guided synergistic therapy in vivo (Figure 4). 
The photothermal heating and release properties of biocompatible organic nanoparticles, doped with a near infrared croconaine (Croc) dye, were compared with analogous nanoparticles doped with the common near-infrared dyes ICG and IR780. Separate formulations of lipid-polymer hybrid nanoparticles and liposomes, each containing Croc dye, absorbed strongly at $808 \mathrm{~nm}$ and generated clean laser-induced heating (no production of 102 and no photobleaching of the dye). In contrast, laser-induced heating of nanoparticles containing ICG or IR780 produced reactive 102, leading to bleaching of the dye and also decomposition of coencapsulated payload such as the drug doxorubicin. Croc dye was especially useful as a photothermal agent for laser-controlled release of chemically sensitive payload from nanoparticles. Solution state experiments demonstrated repetitive fractional release of water-soluble fluorescent dye from the interior of thermosensitive liposomes. Additional experiments used a focused laser beam to control leakage from immobilized liposomes with very high spatial and temporal precision. The results indicate that fractional photothermal leakage from nanoparticles doped with Croc dye is a promising method for a range of controlled release applications [34] (Figure 5).

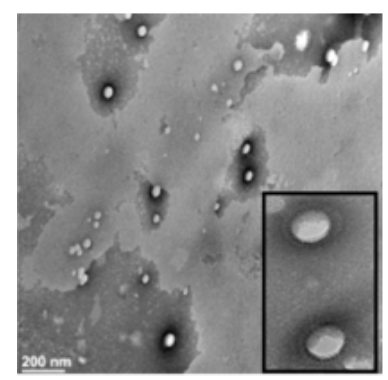

Figure 5: TEM image of Croc/LP-hybrid-NP.

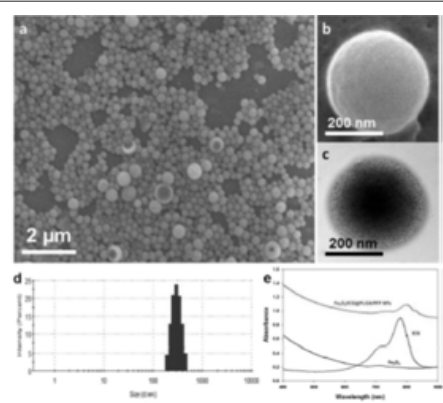

Figure 6: (a,b) SEM images of Fe3O4/ICG@PLGA/PFP NPs (a) 10000x; scale bar, $2 \mu \mathrm{m}$; (b) 70000x; scale bar, $200 \mathrm{~nm}$ ); (c) TEM image of Fe3O4/ICG@PLGA/PFP NPs with a large amount of black Fe3O4 NPs embedded in the spherical shell (70000x; scale bar, 200 nm); (d) size distribution of Fe3O4/ICG@PLGA/PFP NPs with the average diameter of $289.6 \pm 67.4 \mathrm{~nm}$; (e) UV-Vis-NIR absorption spectra of Fe3O4/ICG@PLGA/PFP NPs, free ICG and Fe3O4 NPs.

Near-infrared (NIR) laser-induced photothermal therapy (PTT) uses a photothermal agent to convert optical energy into thermal energy and has great potential as an effective local, minimally invasive treatment modality for killing cancer cells. To improve the efficacy of PTT, Chengcheng Niu et al. [35] developed poly (lactide co-glycolide) (PLGA) nanoparticles (NPs) encapsulating superparamagnetic iron oxide (Fe304), indocyanine green (ICG), and perfluoropentane (PFP) as synergistic agents for NIR laser-induced PTT.
They fabricated a novel type of phase-shifting fluorescent magnetic NPs, Fe304/ICG@PLGA/PFP NPs, that effectively produce heat in response to NIR laser irradiation for an enhanced thermal ablation effect and a phase-shift thermoelastic expansion effect, and thus, can be used as a photothermal agent (Figure 6).

After in vitro treatment of MCF-7 breast cancer cells with Fe304/ICG@PLGA/PFP NPs and NIR laser irradiation, histology and electron microscopy confirmed severe damage to the cells and the formation of many microbubbles with iron particles at the edge or outside of the microbubbles. In vivo experiments in mice with MCF-7 tumors demonstrated that Fe304/ICG@PLGA/PFP NPs could achieve tumor ablation upon NIR laser irradiation with minimal toxicity to non-irradiated tissues. Together, their results indicate that Fe304/ICG@PLGA/PFP NPs can be used as effective nanotheranostic agents for tumor ablation. Hrebesh M. Subhash, et al. [36] have been described a functional imaging paradigm that uses photothermal optical coherence tomography (PT-OCT) to detect indocyanine green (ICG)-encapsulated biocompatible poly(lactic-co-glycolic) acid (PLGA) nanoparticles embedded in highly scattering tissue phantoms with high resolution and sensitivity. The ICG-loaded PLGA nanoparticles were fabricated using a modified emulsification solvent diffusion method.

With a $20 \mathrm{kHz}$ axial scan rate, PT-OCT based on spectral-domain interferometric configuration at $1310 \mathrm{~nm}$ was used to detect phase changes induced by an 808nm photothermal excitation of ICG-encapsulated PLGA nanoparticles. An algorithm based on Fourier transform analysis of differential phase of the spectral interferogram was developed for detecting the depth resolved localized photothermal signal. Excellent contrast difference was observed with PT-OCT between phantoms containing different concentrations of ICG-encapsulated PLGA nanoparticles. This technique has the potential to provide simultaneous structural and molecular-targeted imaging with excellent signal-to-noise for various clinical applications. Photoacoustic imaging (PAI) is an emerging biomedical imaging technique that is now coming to the clinic. It has a penetration depth of a few centimeters and generates useful endogenous contrast, particularly from melanin and oxy-/deoxyhemoglobin. Indocyanine green (ICG) is a Food and Drug Administration-approved contrast agents for human applications, which can be also used in PAI.

It is a small molecule dye with limited applications due to its fast clearance, rapid protein binding, and bleaching effect. Edyta Swider et al. [37] showed that they can label primary human dendritic cells with the nanoparticles and image them in vitro and in vivo, in a multimodal manner. This work demonstrates the potential of combining PAI and 19F MRI for cell imaging and lymph node detection using nanoparticles that are currently produced at GMPgrade for clinical use. Photodynamic therapy (PDT) is emerging as a noninvasive modality for a variety of cancer treatments [38,39]. In typical PDT, a photosensitizer is activated by external light to produce reactive oxygen species (ROS), which can damage cancer cells [40-42]. For deep-seated tumors, the efficiency of PDT is decreased due to the limited penetration depth of external light in biological tissue. This problem has hindered further development of PDT in 
widespread clinical applications. In addition, skin photosensitivity and photothermal injury are also common concerns from the patients receiving PDT treatment [43-45].

For the light penetration issue to be overcome, considerable research has focused on the development of photosensitizers in the near infrared (NIR) range [46]. Up-conversion nanoparticles combined with photosensitizers have been explored because they can absorb NIR photons and emit visible light to activate the photosensitizer for PDT $[47,48]$. Researchers have explored new approaches, for example, using Cerenkov radiation to activate a photosensitizer for effective PDT $[49,50]$. Alternatively, X-ray is currently widely used in clinical treatment, and X-ray-activated photodynamic therapy is another way to overcome the light penetration limitation in deep-seated tumors [51-53]. The use of an internal light source is an intriguing solution for the light-penetration problem. For example, implanting fiber-optic light sources and emitting diodes could be a viable approach, but it still requires invasive operation [54-56]. Alternatively, bioluminescence offers an attractive strategy because of the minimally invasive delivery of enzymes and substrates.

Bioluminescence is a natural phenomenon that occurs in various organisms, such as marine organisms and some insects, in which visible light is produced via chemical reactions in vivo $[57,58]$. Bioluminescence has been widely used in biological detection and optical imaging [59-61]. Recently, researchers have recognized the therapeutic potential of bioluminescence. Bioluminescence is endogenous fluorescence that can be used to activate the photosensitizer inside the tumors. This process is not affected by the light penetration depth of tissue. Wang et al. [62] reported that luminol has anticancer and antifungal activities through bioluminescence resonance energy transfer (BRET) [62]. Moreover, quantum dots have also been combined with the BRET system for in vivo imaging and PDT in recent reports [63-65]. Despite these progresses, the BRET-activated ROS generation and photodynamic effect is largely unexplored. They show effective photodynamic therapy mediated by the firefly luciferase bioluminescence system. Biodegradable poly (lactic-co-glycolic acid) (PLGA) nanoparticles were doped with the photosensitizer Rose Bengal (RB).

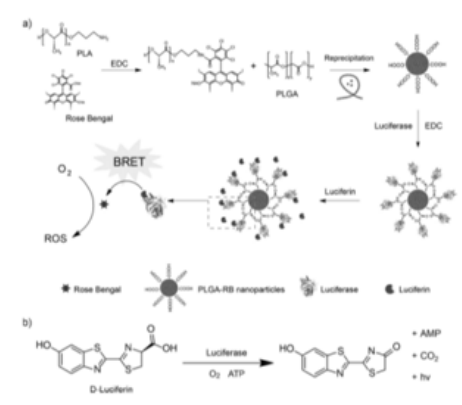

Figure 7: (a) Schematic illustration of the preparation of PLGA-RB nanoparticles. (b) Firetly luciferase catalyzing the oxidation reaction of the native substrate D-luciferin for photon emission.

The PLGA-RB nanoparticles were then conjugated with luciferase, which produced a fluorescent signal in the presence of the luciferin substrate. The endogenous bioluminescence served as a light source to activate the photosensitizer for ROS generation.
They evaluated cell toxicity, cellular fluorescence imaging, and therapeutic effect in cellular assays, which indicated that cancer cells were destroyed effectively. The antitumor effect of BRET-PDT was further investigated in tumor-bearing ICR mice, which showed that tumor growth was significantly inhibited. This study provides a promising approach for phototherapy of deep-seated tumors in the absence of external excitation (Figure 7).

Nanoparticles present great potential in drug delivery applications, yet there are some issues regarding their stability. In this study was conducted to define the conditions to stabilize polysaccharide (chitosan/dextran sulfate, CS/DS) nanoparticles by a process of freeze-drying, assessing the cryoprotectant capacity of two sugars (sucrose and glucose). Additionally, it was also intended to find if the solubilisation of chitosan in different acids affected nanoparticle preparation and characteristics. Chitosan is obtained by the N-deacetylation of chitin, a polymer that can be extracted from various sources (crustacean's shells, exoskeletons of certain invertebrates - ladybugs - and the cell walls of fungi, for example). It is considered the second most abundant polymer on Earth [66], after cellulose [67]. However, chitin is not very versatile due to its structure and poor solubility in many solvents. Chitin plays a role in the protection of certain animals in nature, being organized in semi-crystalline microfibrils to provide the said protection [68]. The deacetylated form of chitin is chitosan, which structure can be seen on (Figure 8).

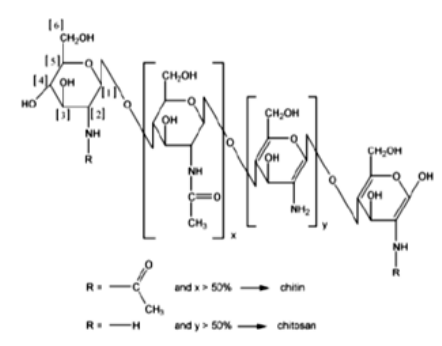

Figure 8: Chemical structure of Chitin and Chitosan.

Chitosan (MW $\sim 30-190 \mathrm{kDa}$ ) is the polymer obtained when deacetylation surpasses $50 \%$ and is comprised of $\beta$ (1-4)-links of $\mathrm{D}$-glucosamine and $\mathrm{N}$-acetyl-D-glucosamine monomers that are distributed randomly throughout the chain. This is the only natural polymer exhibiting a cationic character [69]. Due to the fact that the amine groups are present throughout the structure of chitosan, the more deacetylated the polymer is, the more susceptible it is to protonation because nitrogen has an unused pair of electrons that can easily interact with electrophilic groups. When chitosan undergoes protonation, which occurs at low $\mathrm{pH}$ levels, it acquires a positive charge, thus providing the possibility to interact with negatively charged groups. This ability has been widely explored in drug delivery, with the preparation of nanoparticles by electrostatic interaction, as reported using carrageenan [70], tripolyphosphate (TPP) or dextran sulfate [71] as counterions. Chitosan is not soluble in water. Instead, and because the amine groups have a logarithmic acidity constant (pKa) of $\sim 6.5$, chitosan dissolves easily in acidic media. The most usual solvent for its dissolution is $1 \%(\mathrm{v} / \mathrm{v})$ acetic acid. 
Due to the fact that the polymer is easily protonated, it can also be dissolved in formic and lactic acids and in hydrochloric acid solutions [72]. In a pH of 7 or higher, as the $\mathrm{pH}$ is higher than the pKa of amine groups, chitosan becomes insoluble, rendering its biologically applications are scarce or non-existent. As mentioned earlier, chitosan presents adequate characteristics regarding biological applications [73], including biocompatibility, biodegradability and low toxicity. Its biodegradability is due to a metabolism by lysozyme [74]. Moreover, due to its protonation in acidic media, it presents a favourable interaction with mucus [75], which is particularly relevant in the intestine, where there is a high amount of mucus. This is actually one of the reasons justifying its application in specific disease conditions, such as colonic inflammation. Nevertheless, the description of chitosan applications in literature is endless, including pulmonary and oral insulin delivery as well as the delivery of antibiotics and small genetic fragments [76-78], just to mention some examples. Finally, chitosan can also be found in a variety of dietary supplements (Lipoforte ${ }^{\circledR}$ and EasySlim $\AA$ Blocker, just to name a few) for weight loss.

However, the European Food Safety Authority (EFSA) considers that there is not enough evidence that supports this indication. There are other studies that report its beneficial use on lowering LDL-cholesterol $[79,80]$, a claim that EFSA found to have enough evidence that supports it. However, some problems have also been described for supplements, reporting chitosan interference with certain treatments, for example, anticoagulation therapy with warfarin [81] and anti-epilepsy therapy with valproate [82]. Dextran sulfate was the other polymer used in this experimental work. It is also a natural polymer, obtained from bacteria of the Leuconostoc genus. It is comprised of a branched anhydroglucose backbone with attached sulphur groups, the latter believed to be $17 \%$ of the total mass of the polymer. This means that, 2.3 sulphur groups are present for each glucosyl residue, thus the polymer being expected to be negatively charged. Its chemical structure can be seen in Figure 2. Dextran sulfate (MW $\sim 5-500 \mathrm{kDa}$ [83-85] is also reported as biocompatible and biodegradable [86], thus theoretically complying with mandatory requisites for biomedical applications.

Studies regarding the use of dextran sulfate as a component of DDS are scarce, even though it was widely studied in the eighties for its anti-HIV activity, against HIV-1. Its mechanism of action, in this context, involves inhibiting the reverse transcriptase, a viral enzyme responsible for turning the viral RNA in viral DNA that will, ultimately, be part of the host cell DNA resulting in the production of viral particles [87]. Additionally, dextran sulfate has also been shown to activate macrophages by establishing an interaction with the scavenger receptor present in these cells [88]. Moreover, regarding the immune system, it is believed that dextran sulfate induces activation of $B$ lymphocytes even though that mechanism is mediated by macrophages [89]. Another aspect that is widely mentioned in studies is an anticoagulant capacity similar to heparin, even though it wasn't studied further [90]. Finally, dextran sulfate is used, nowadays, to induce colitis in mouse models [91,92]. Approved formulations containing this polymer include two EMA-approved orphan medicines: a) a medicine for the prevention of graft rejection and its further damage on pancreatic transplantation by inhibiting the activation of complement system and clotting [93]

b) a medicine to promote mobilisation of progenitor cells, prior to stem cell transplantation, through a cytokine released by bone marrow that can guide these important cells through blood stream [94].

Dextran sulfate is a very interesting polymer with many characteristics that can be used in drug delivery. However, there is still much work to do and, more importantly, study possible associations with other polymers to produce nanocarriers with a potential use in therapeutics [95-97]. Chitosan extracted from Metacarcinus magister shells using demineralization with $1.5 \mathrm{M}$ and $2 \mathrm{M} \mathrm{HCl}$ showed the characteristic functional groups of CS. The CS-CMC nanoparticle were chelated with five different concentrations of TPP and $\mathrm{BaCl} 2$ $(0.1 \%, 0.2 \%, 0.3 \%, 0.4 \%$ and $0.5 \%)$ and embodied with CUR. The SEM analysis indicated the size of the nanoparticles to below 500 $\mathrm{nm}$ which was also confirmed by AFM. The encapsulation efficiency was absolutely time dependent. The drug release was found to be best with higher concentration of chelating agents used and the drug release was prolonging to 80th minute for all the samples. In vitro drug release showed antibacterial activity when ethanol and acetic acid was used as solvent system [98]. A new granule hydrogel composites of natural biocompatible polymeric chitosan and gelatin (C-G) have prepared. The gelatin side groups (hydroxyl and carboxyl) can attach with amine groups of chitosan, which improve the interlink crosslinking in the final nanocomposites.

The diameter of the granuls is around 3mm to $6 \mathrm{~mm}$. Furthermore, in order to reinforce the mechanical properties of granules, for the first time, octa (ammonium chloride) substituted Polyhedral Oligomeric Silsesquioxane C-G/POSS-(NH3+ $\mathrm{Cl}-)_{8} 8$ has been used as a nanofiller in the structure of this nanocomposite. The effect of C-G/POSS-(NH3+ Cl-)8 on mechanical stability and swelling behavior of hydrogels has been investigated in three $\mathrm{pH}$ of 1.2, 7.4 and 9.5, respectively. Results demonstrate that the C-G/POSS$(\mathrm{NH} 3+\mathrm{Cl}-) 8$ granules in acidic $\mathrm{pH}$ has the maximum swelling percent. The encapsulation efficiency (EE) values for C-G granules in three $\mathrm{pH}$ of 1.2, 7.4 and 9.5 are obtained $65 \%, 58 \%$ and $53 \%$ and for C-G/POSS-(NH3+ Cl-) 8 sample are 59\%, 52\% and 48\%, respectively, which conform with the swelling behavior of these hydrogels. According to the obtained results from Metronidazole (MTZ) release, the as-synthesized hydrogels may have intrinsic ability in the controlled release of drugs. The as-prepared hydrogels are characterized via FT-IR, SEM, TGA and XRD analysis [99]. Chitosan nanoparticles (CS NPs) exhibit good physicochemical properties as drug delivery systems. Haliza Katas et al. [100] have determined the modulation of preparative parameters on the physical characteristics and colloidal stability of CS NPs. CS NPs were fabricated by ionic interaction with dextran sulphate (DS) prior to determination of their storage stability.

The smallest CS NPs of $353 \pm 23 \mathrm{~nm}$ with a surface charge of $+56.2 \pm 1.5 \mathrm{mV}$ were produced when CS and DS were mixed at $\mathrm{pH} 4$ and with a DS : CS mass ratio of $0.5: 1$. An entrapment efficiency of 
98\% was achieved when BSA/siRNA was loaded into the nanoparticles. The results also showed that particle size and surface charge of CS NPs were slightly changed up to 2 weeks when stored at 40C. Greater particle size and surface charge were obtained with increasing the concentration of DS. In conclusion, NPs were sufficiently stable when kept at $4 \circ \mathrm{C}$ and able to carry and protect protein. Ji Woon Suh et al. [101] have formulated buccal mucoadhesive nanoparticles (NPs) using the natural mucoadhesive polymers. The natural mucoadhesive polymers chitosan (CS) and dextran sulfate sodium salt (DS) were used to prepare mucoadhesive NPs using the ionic gelation method. As the molecular weight of DS decreased, the amount of mucin and the number of buccal cells adsorbed on DS increased. The CS/DS NPs ranged from 100 to $200 \mathrm{~nm}$ in diameter.

The adhesive interactions of CS/DS NPs with mucin were not significantly different from those of CS/sodium triphosphate pentabasic (TPP) NPs; however, CS/DS NPs exhibited 5 times greater mucoadhesive activity to buccal cells compared to control CS/TPP NPs in ex vivo adhesion tests. These results indicate that the buccal mucoadhesive properties of NPs can be improved using natural mucoadhesive polymers. A novel nanoparticle delivery system has been developed by employing the oppositely charged polymers chitosan (CS) and dextran sulfate (DS), and a simple coacervation process. Under the conditions investigated, the weight ratio of the two polymers is identified as a determining factor controlling particle size, surface charge, entrapment efficiency and release characteristics of the nanoparticles produced. Particles of $223 \mathrm{~nm}$ mean diameter were produced under optimal conditions with a zeta potential of approximately $-32.6 \mathrm{mV}$. A maximum of $75 \%$ anti-angiogenesis peptide entrapment efficiency was achieved with a CS:DS weight ratio of 0.59:1. The same nanoparticle formulation also showed slow and sustained peptide release over a period of 6 days.

In contrast, the formulation containing a lower ratio of CS:DS (0.5:1) was found to have reduced entrapment efficiency and more rapid peptide release characteristics. The results suggest that physicochemical and release characteristics of the CS-DS nanoparticles can be modulated by changing ratios of two ionic polymers. The novel CS-DS nanoparticles prepared by the coacervation process have potential as a carrier for small peptides [102]. Fwu-Long Mi [40] have prepared chitosan-based nanoparticles containing indocyanine green (ICG) for hyperthermia therapy and imaging. ICG was incorporated into chitosan and poly-gamma-glutamic acid self-assembled nanoparticles using the polyelectrolyte complex method. The ICG encapsulation efficiency of the nanoparticles were higher than $95 \%$ and the ICG-loaded nanoparticles showed effective temperature elevation upon NIR laser irradiation $(808 \mathrm{~nm}, 1.5 \mathrm{~W} / \mathrm{cm} 2)$. The cytotoxicity results from NIR laser-induced hyperthermia effect showed an enhanced toxicity in overcoming doxorubicin (Dox) resistance of MCF-7/ADR cells.

Pei-Ru Wei et al. [103] have designed a study for photodynamic therapy (PDT) using chitosan coated Mg-Al layered double hydroxide (LDH) nanoparticles as the delivery system. A Food and Drug Administration (FDA) approved near-infrared (NIR) fluorescent dye, indocyanine green (ICG) with photoactive properties was intercalated into amine modified LDH interlayers by ion-exchange.
The efficient positively charged polymer (chitosan (CS)) coating was achieved by the cross linkage using surface amine groups modified on the LDH nanoparticle surface with glutaraldehyde as a spacer. The unique hybridization of organic-inorganic nanocomposites rendered more effective and successful photodynamic therapy due to the photosensitizer stabilization in the interlayer of LDH, which prevents the leaching and metabolization of the photosensitizer in the physiological conditions. The results indicated that the polymer coating and the number of polymer coats have a significant impact on the photo-toxicity of the nano-composites [104-106]. The double layer chitosan coated LDH-NH2-ICG nanoparticles exhibited enhanced photo therapeutic effect compared with uncoated LDH-NH2-ICG and single layer chitosan-coated LDH-NH2-ICG due to the enhanced protection to photosensitizers against photo and thermal degradations.

This new class of organic-inorganic hybrid nanocomposites can potentially serve as a platform for future non-invasive cancer diagnosis and therapy. Nanoparticles (NPs) are synthetic materials with dimensions from one to hundreds of nanometers, and remarkable applications in biomedicine due to the unique way in which they interact with matter $[107,108]$. There are currently more than 35 US FDA-approved NPs often incorporating polyethylene glycol (PEG), with a larger number in preclinical studies for both imaging and therapy (Figure 9A) [109-115]. NPs have large payloads, stability, avidity, signal enhancement and the capacity for multiple, simultaneous applications owing to their unique size and high surface area: volume ratio [116]. While they are bigger than molecules and many proteins, yet smaller than cells, they behave differently to other therapies and imaging agents, affecting their in vivo applications. For example, in cancer tissue, NPs not only extrava-sate from the leaky tumor vasculature to a higher degree than healthy tissue, but also remain in the area by the enhanced permeability and retention (EPR) effect [118].

NPs lodged in the tumor can then perform signaling and/or therapy [117]. Despite these advantages, some fundamental challenges hamper NP deployment to the clinic. These include uptake by the reticuloendothelial system (RES), in which NPs are rapidly shuttled out of circulation to the liver, spleen or bone marrow, and nonspecific binding of NPs to nontargeted or nondiseased areas. Concerns about NP toxicity often arise because of this RES accumulation. Aggregation can lead to NP entrapment in the liver, lungs or elsewhere due to capillary occlusion [119]. The addition of PEG to the NP surface (PEGylation) can reduce many of these challenges (Figure 1B). PEG is a coiled polymer of repeating ethylene ether units with dynamic conformations (Figure 1C). In both drug-delivery and imaging applications, the addition of PEG to NPs reduces RES uptake and increases circulation time versus uncoated counterparts [120]. Aggregation decreases owing to passivated surfaces, and association with nontargeted serum and tissue proteins is diminished, resulting in so-called 'stealth' behavior.

The PEG chains reduce the charge-based contact typical of proteins and small-molecule interactions. Solubility in buffer and serum increases due to the hydrophilic ethylene glycol repeats and the EPR effect is modulated due to NP size changes via addition 
of a PEG coat $[121,122]$. Due to these attributes, PEGylated NPs generally accumulate in the liver a half to a third of the amount of non-PEGylated NPs and demonstrate higher tumor accumulation versus background [123]. PEG is inexpensive, versatile and FDA approved for many applications [124]. All NPs contain at least two fundamental spatial components: the core and the corona that interact with the environment or solvent. While core/shell, core/ multishell systems add further complexity, for example [125], all still possess an area in which NP interfaces with the solvent (Figure 9B). PEG chains modify this interface layer and increase circulation time. Circulation half-time $\left(\mathrm{t}^{1} / 2\right)$ describes blood pool residence and is the period over which the concentration of circulating NPs remains above $50 \%$ of the injected dose, analogous to a drug's halflife [126].

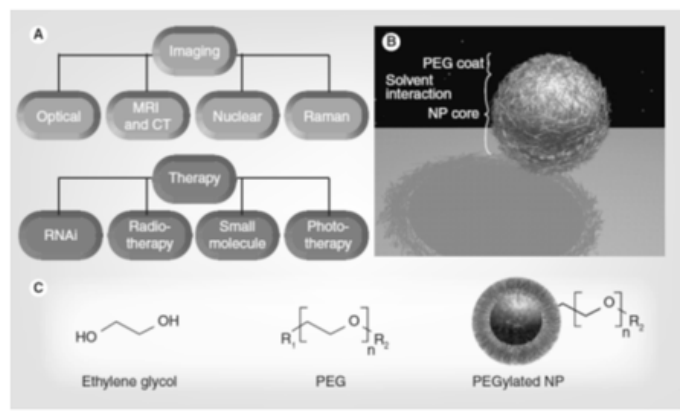

Figure 9: Nanoparticle applications of Polyethylene glycol.

NP efficacy requires sufficient $\mathrm{t}^{1} / 2$ to not only reach the target, but also remain in the affected area (at concentrations sufficiently above background tissue) long enough for image capture or drug delivery. The RES system prevents site-specific accumulation because it removes the NPs from circulation, acting as a competitor to the intended target site [127]. In addition, the NPs must clear from the nontargeted area to produce imaging contrast or dosing efficiency. The ideal $t \frac{1}{2}$ is dependent on application. In imaging, 2-6 $\mathrm{h}$ is optimal for injection, accumulation at targeted site, clearance from nontargeted areas and data collection. The ideal circulation time for therapeutic NPs is longer (days) to allow repeated exposure to affected area. Unfortunately, this can also expose healthy organ systems to the drug and is the motivation for targeted NPs, as such systems preferentially accumulate in the diseased area. Approaches to measuring $t^{1} 1 / 2$ vary with NP type. When labeled with radionuclides, g counting of either specific organ systems or blood aliquots determines NP circulation time. One limitation is dissociation of radionuclide from NPs; however, radioactivity measurements may always be carried out noninvasively [128] (Figure 9).

Measurement of $t^{1} / 2$ via fluorescence, Raman, inductively coupled plasma or chromatography/mass spectrometry is very specific to the NP, but requires sequential sampling of the blood pool. The RES is an immune system component, utilizing circulating macrophages and monocytes, liver Kupffer cells and spleen and other lymphatic vessels to remove foreign material, such as bacteria and viruses, from the body [127]. (Figure 10) illustrates how opsonin proteins associate with foreign bodies and coat its surface [128]. As bacteria and viruses have the same negative surface charge as phagocytic cells, opsonins are critical to reducing the charge re- pulsion between the two systems. Next, phagocytic cells engulf the material and transport it to the liver or spleen for degradation and excretion (Figure 10A3-A4). Additional phagocytic macrophages are permanently located in the liver. Known as Kupffer cells, these cells serve as a major filter for many types of NPs and are a major interference with long $t^{1} / 2$ [129]. The PEG polymer on a NP surface increases $t \frac{1}{2}$ by reducing this opsonization process (Figure 10B2), thus preventing recognition by monocytes and macrophages, allowing the NPs to remain in the blood pool.

Hydrophobic particles are also more vulnerable to the RES and hydrophilic PEG reduces these complications. In addition to NPRES interactions, poor $\mathrm{t}^{1} / 2$ can also result from NP-NP interactions (i.e., aggregation). NPs aggregate primarily because the attraction between particles is stronger than the attraction for solvent [130]. NPs with a high surface energy have a greater tendency to aggregate as described by the Derjaguin-Landau- Verwey-Overbeek (DLVO) theory $[131,132]$. For spherical NPs, the interaction potential is related to the electrostatic repulsive potential and the van der Waals attraction potential [133]. PEG decreases the surface energy of NPs and minimizes van der Waals attraction $[134,135]$. Aggregation can be induced by solvents of high $(>100 \mathrm{mM})$ ionic strength (shielding of sol $\neg$ vent from NP), highly concentrated solutions of NPs (less distance between the NPs), time from synthesis, or NP preparations with a very neutral $(\sim \pm 5 \mathrm{mV})$ zeta potential [136]. PEG decreases the amount of attraction between NPs by increasing the steric distance between them and increasing hydrophilicity via ether repeats forming hydrogen bonds with solvent. Other benefits to PEGylation include modifying the size of the particle. The reduced renal filtration of particles larger than $10 \mathrm{~nm}$ increases $\mathrm{t} 1 / 2$; however, at too large a size $(>100 \mathrm{~nm})$, liver uptake increases and EPR extravasation may decrease [137]. PEG modifies the NP flexibility and the NP can become 'softer' after PEGylation than the underlying material, influencing extravasation.

Prior to NP applications, PEG was used as a nontoxic, water-soluble dispersant/stabilizer. Also known as Carboxwax®, it is present in health and beauty aids, including laxatives, toothpastes and eye drops, and is an excipient in tablet formulations [138]. PEG stabilizes organ and blood donations. Early work with PEGylated NPs stemmed mostly from drug delivery [139]. One of the first reports on PEGylation was described by Davis and Abuchowski $[140,141]$, where they covalently attached methoxy-PEGs (mPEGs) of 1900 and 5000 Da to bovine serum albumin and to liver catalase. Later, acrylic microspheres functionalized with PEG-modified human serum albumin increased $t \frac{1}{2}$ in vivo [142]. Li and colleagues found that 75-nm latex particles remained in rat circulation 40-times longer (half-life $20 \mathrm{~min}$ vs $13 \mathrm{~h}$ ) when coated than uncoated with PEG larger than $5000 \mathrm{kDa}$ [143]. Klibanov and Huang found that incorporation of dioleoyl $\mathrm{N}$-(monomethoxy polyethyleneglycol succinyl) phosphotidylethanolamine (PEG-PE) into posphatidylcholine: cholesterol liposomes $(1: 1)$ increased $t^{1} / 2$ from 30 min to $5 \mathrm{~h}$ without increasing leakage of the liposome interior. In the mid-1990s, Doxil ${ }^{\circledR}$ (liposomal delivery vehicle for doxorubicin) and oncospar (PEG-l-asparaginase) became the first FDA-approved NP therapeutics [143]. Doxil increases doxorubicin bioavailability nearly 90- 
fold at 1 week from injection of PEGylated liposomes versus free drug [144].

The use of PEG on the doxorubicin carrier yields a drug half-life of $72 \mathrm{~h}$ with circulation half-life of $36 \mathrm{~h}[145,146]$. Later, Abraxane ${ }^{\circledR}$ was introduced as an albumin-functionalized NP for delivery of taxane without cremphor to enhance drug efficiency. Thadakapally et al. [151] developed a novel serum stable long circulating polymeric nanoparticles for curcumin with a modification to the well-known and novel nanoparticle albumin bound technology. polyethylene glycolalbumin-curcumin nanoparticles were prepared using serum albumin and poly ethylene glycol using desolvation technique. Nanoparticles were characterized for encapsulation efficiency, particle size and surface morphology. Drug excipient compatibility was determined using fourier transform infrared spectroscopy. Physical state of the drug in the formulations was known by differential scanning colorimetry. In vitro release and solubility of the drug from nanoparticles were determined. In vivo Drug release, tissue uptake and kupffer cell uptake was determined with optimized nano formulation in rats after intravenous administration. Cell viability assay was determined using breast cancer cell line MD-MB-231.

Entrapment efficiency for prepared nanoparticle was above 95\%. The polyethylene glycol-albumin-curcumin nanoparticles exhibited an interesting release profile with small initial burst followed by slower and controlled release. Solubility of the drug from the formulation was increased. A sustained release of drug from nanoparticles was observed for 35 days in both in vitro and in vivo studies with the optimized formulation. Polyethylene glycol-albumin-curcumin nanoparticles showed lesser liver and kupffer cell uptake as compared to that of curcumin-albumin nanoparticles suggesting the bestowment of stealthness to nanoparticles with pegylation. Also, the antiproliferative activity of polyethylene glycol-albumin-curcumin nanoparticle formulation was more as compared to native curcumin. Polyethylene glycol-albumin-curcumin nanoparticles thus developed can be conveniently used in breast cancer with improved efficacy compared to conventional therapies and as an alternate to nanoparticle albumin bound technology which is used in producing Abraxane, albumin based breast cancer targeting nanoparticles of paclitaxel [147].

Photothermal therapy (PTT) and photodynamic therapy (PDT) are emerging physical tumor treatments utilizing near infrared (NIR) light-absorbing agents which lead to thermal ablation of cancer cells or generate highly reactive oxygen species (ROS) via photosensitizer to ablate tumors [148,149]. PTT and PDT possess several advantages, such as minimal invasion, high therapeutic efficacy, limited side-effects, selective localized treatment and reproducible properties $[150,151]$, and hence have received much attention in recent years $[152,153]$. Until now, a variety of materials has been explored as PTT or PDT agents due to their high absorption in the tissue-transparent NIR wavelength range, including organic fluorescent dyes [154], gold nanorods [155], CuS nanoparticles (NPs) [156], polymer NPs [157], carbon nanomaterials [158], etc. $[159,160]$. However, fluorescent dyes may be removed rapidly from the systemic circulation and lack specificity to a tumor, and inor- ganic photothermal agents have potential long-term toxicity due to the difficulty of degrading in the body [161].

Therefore, exploiting biocompatible and targeted therapeutic nanoagents with enhanced photothermal conversion capability and ROS generation ability to amplify PTT and PDT treatments remains challenging. Indocyanine green (ICG) is a clinical infrared imaging agent approved by the U.S. Food and Drug Administration (FDA), and has been applied in optical imaging of human vasculature, tissue and cells due to its biocompatibility and unique optical properties [162]. Due to strong absorption at 780nm, ICG can effectively convert absorbed NIR optical energy into heat for PTT [163] and produce ROS for PDT [164] under NIR laser irradiation [165]. Nevertheless, the application of ICG in tumor phototherapy is limited by its tendency to aggregate, rapid degradation in aqueous solution [64], poor photo-stability and non-specific binding to proteins [166]. To overcome those limitations, various nanoparticle delivery systems have been developed to encapsulate ICG [167]. Lv et al. [168] used a mesoporous silica (mSiO2) matrix to load ICG molecules and demonstrated that loaded ICG displayed a more enhanced photothermal effect than pure ICG [168]. ICG-loaded mesoporous silica NPs also could not only limit the degradation of ICG but reach and stay at a tumor for a long period of time due to an enhanced permeability and retention (EPR) effect [169]. Hence, loading of ICG within targeting nanocarriers with high efficiency is shown to be an effective way to promote the application of ICG in PTT and PDT treatment.

\section{Conclusion}

Multifunctional biodegradable and biocompatible polymeric nanoparticles loaded with indocyanine green (ICG) as an optical-imaging contrast agent for cancer imaging and as a photothermal therapy agent for cancer treatment. To improve the efficacy of PTT, polymeric nanoparticles (NPs) encapsulating superparamagnetic iron oxide (Fe304), indocyanine green (ICG), and perfluoropentane (PFP) as synergistic agents for NIR laser-induced PTT.

\section{References}

1. Cheng L, Wang C, Feng LZ, Yang K, Liu Z (2014) Functional nanomaterials for phototherapies of cancer. Chem Rev 114(21): 10869-10939.

2. Dolmans DE, Fukumura D, Jain RK (2003) Photodynamic therapy for cancer. Nat Rev Cancer 3(5): 380-387.

3. Circu ML, AW TY (2010) Reactive oxygen species, cellular redox systems, and apoptosis. Free Radic Biol Med 48(6): 749-762.

4. Schaafsma BE, Mieog JS, Hutteman M, van der Vorst JR, Kuppen PJ, et al. (2011) The clinical use of indocyanine green as a near-infrared fluorescent contrast agent for image-guided oncologic surgery. J Surg Oncol 104(3): 323-332.

5. Mastropasqua R, Di Antonio L, Di Staso S, Agnifili L, Di Gregorio A, et al. (2015) Optical Coherence Tomography Angiography in Retinal Vascular Diseases and Choroidal Neovascularization. J Ophthalmol 343515.

6. Zelken JA, Tufaro AP (2015) Current Trends and Emerging Future of Indocyanine Green Usage in Surgery and Oncology: An Update. Ann Surg Oncol 3: S1271-S1283.

7. Shemesh CS, Moshkelani D, Zhang H (2015) Thermosensitive liposome formulated indocyanine green for near-infrared triggered photodynamic therapy: in vivo evaluation for triple-negative breast cancer. Pharm Res 32(5): 1604-1614. 
8. Bernardi RJ, Lowery AR, Thompson PA, Blaney SM, West JL (2008) Immunonanoshells for targeted photothermal ablation in medulloblastoma and glioma: an in vitro evaluation using human cell lines. J Neurooncol 86(2): 165-172.

9. Mundra V, Peng Y, Rana S, Natarajan A, Mahato RI (2015) Micellar formulation of indocyanine green for phototherapy of melanoma. J Control Release 220: 130-140.

10. Saxena V, Sadoqi M, Shao J (2003) Degradation kinetics of indocyanine green in aqueous solution. J Pharm Sci 92(10): 2090-2097.

11. Mordon S, Devoisselle JM, Soulie Begu S, Desmettre T (1998) Indocyanine green: physicochemical factors affecting its fluorescence in vivo. Microvasc Res 55(2): 146-152.

12. Desmettre T, Devoisselle JM, Mordon S (2000) Fluorescence properties and metabolic features of indocyanine green (ICG) as related to angiography. Surv Ophthalmol 45(1): 15-27.

13. Jain KK (2008) Nanomedicine: application of nanobiotechnology in medical practice. Med Princ Pract 17(2): 89-101.

14. Nair LS, Laurencin CT (2007) Biodegradable polymers as biomaterials. Prog Polym Sci 32(8-9): 762-798.

15. Makadia HK, Siegel SJ (2011) Poly lactic-co-glycolic acid (PLGA) as biodegradable controlled drug delivery carrier. Polymers 3(3): 13771397.

16. Fishburn CS (2008) The pharmacology of PEGylation: Balancing PD with PK to generate novel therapeutics. J Pharm Sci 97(10): 4167-4183.

17. BjoÈrnsson OG, Murphy R, Chadwick VS, BjoÈrnsson S (1983) Physiochemical studies on indocyanine green: molar lineic absorbance, $\mathrm{pH}$ tolerance, activation energy and rate of decay in various solvents. J Clin Chem Clin Biochem 21(7): 453-458.

18. Saxena V, Sadoqi M, Shao J (2004) Enhanced photo-stability, thermalstability and aqueous-stability of indocyanine green in polymeric nanoparticulate systems. J Photochem Photobiol B 74(1): 29-38.

19. Yaseen MA, Yu J, Wong MS, Anvari Bn (2007) Stability assessment of indocyanine green within dextran-coated mesocapsules by absorbance spectroscopy. J Biomed Opt 12(6): 064031.

20. Zheng CF, Zheng MB, Gong P, Jia AG, Zhang PF, et al. (2012) Indocyanine green-loaded biodegradable tumor targeting nanoprobes for in vitro and in vivo imaging. Biomaterials 33(22): 5603-5609.

21. Altinoglu EI, Russin TJ, Kaiser JM, Barth BM, Eklund PC, et al. (2008) Near- Infrared Emitting Fluorophore-Doped Calcium Phosphate Nanoparticles for In Vivo Imaging of Human Breast Cancer. ACS Nano 2(10): 2075-2084.

22. Liu H, Farrell S, Uhrich K (2000) Drug release characteristics of unimolecular polymeric micelles. J Controlled Release 68(2): 167-174.

23. Lavasanifar A, Samuel J, Kwon GS (2002) Poly (ethylene oxide)-blockpoly (L-amino acid) micelles for drug delivery. Adv Drug Delivery Rev 54(2): 169-190.

24. Chen YP, Li XD (2011) Near-Infrared Fluorescent Nanocapsules with Reversible Response to Thermal/pH Modulation for Optical Imaging. Biomacromolecules 12(12): 4367-4372.

25. Kim TH, Chen Y, Mount CW, Gombotz WR, Li XD, et al. (2010) Evaluation of Temperature-Sensitive, Indocyanine Green-Encapsulating Micelles for Noninvasive Near-Infrared Tumor Imaging. Pharm Res 27(9): 19001913.

26. Kirchherr AK, Briel A, Mader K (2009) Stabilization of indocyanine green by encapsulation within micellar systems. Mol Pharmaceutics 6(2): 480-491.

27. Zheng XH, Xing D, Zhou FF, Wu BY, Chen, et al. (2011) Indocyanine GreenContaining Nanostructure as Near Infrared Dual-Functional Targeting Probes for Optical Imaging and Photothermal Therapy. Mol Pharm 8(2): 447-456.
28. Zheng XH, Zhou FF, Wu BY, Chen WR, Xing D (2012) Enhanced Tumor Treatment Using Biofunctional Indocyanine Green-Containing Nanostructure by Intratumoral or Intravenous Injection. Mol Pharmaceutics 9(3): 514-522.

29. Romila Manchanda, Tingjun Lei, Yuan Tang, Alicia Fernandez-Fernandez, Anthony J McGoron (2010) Cellular Uptake and Cytotoxicity of a Novel ICG-DOX-PLGA Dual Agent Polymer Nanoparticle Delivery System. In: K E Herold, W E Bentley, J Vossoughi (Eds.), SBEC IFMBE Proceedings 32: 228-231.

30. Takehiro Noda, Tomoyuki Okuda, Ryota Mizuno, Tetsuya Ozeki, Hirokazu Okamoto (2018) Two-Step Sustained-Release PLGA/Hyaluronic Acid Gel Formulation for Intra-articular Administration. Biol Pharm Bull 41(6): 937-943.

31. Ronak H Patel, Aniket S Wadajkar, Nimit L Patel, Venkaiah C Kavuri, Kytai T Nguyen, et al. (2012) Multifunctionality of indocyanine greenloaded biodegradable nanoparticles for enhanced optical imaging and hyperthermia intervention of cancer. Journal of Biomedical Optics 17(4): 046003.

32. Tayebeh Akbari, Maryam Pourhajibagher, Nasim Chiniforush, Sima Shahabi, Farzaneh Hosseini, et al. (2018) Improve ICG Based Photodynamic Properties Through Conjugation of ICG Into NanoGraphene Oxide Against Enterococcus faecalis. Avicenna J Clin Microbiol Infect 5(1): e64624.

33. Bin Zheng, Yang Bai, Hongbin Chen, Huizhuo Pan, Wanying Ji, et al. (2018) Targeted delivery of tungsten oxide nanoparticles for multifunctional anti-tumor therapy via macrophages. Biomaterials Science.

34. Samit Guha, Scott K Shaw, Graeme T Spence, Felicia M Roland, Bradley D Smith (2015) Clean Photothermal Heating and Controlled Release from Near-Infrared Dye Doped Nanoparticles without Oxygen Photosensitizatio. Langmuir 31(28): 7826-7834.

35. Chengcheng Niu, Yan $\mathrm{Xu}$, Senbo An, Ming Zhang, Yihe $\mathrm{Hu}$, et al. (2017) Near-infrared induced phaseshifted ICG/Fe304 loaded PLGA nanoparticles for photothermal tumor ablation. Sci Rep 7(1): 5490.

36. Hrebesh M Subhash, Hui Xie, Jeffrey W Smith, Owen JT McCarty (2012) Optical detection of indocyanine green encapsulated biocompatible poly (lactic-co-glycolic) acid nanoparticles with photothermal optical coherence tomography. Opt Lett 37(5): 981-983.

37. Edyta Swider, Khalid Daoudi, Alexander H J Staal, Olga Koshkina, N Koen van Riessen, et al. (2018) Clinically-Applicable Perfluorocarbon-Loaded Nanoparticles For In vivo Photoacoustic, 19F Magnetic Resonance And Fluorescent Imaging. Nanotheranostics 2(3): 258-268.

38. Dolmans DE, Fukumura D, Jain RK (2003) Photodynamic therapy for cancer. Nat Rev Cancer 3(5): 380-387.

39. Moore CM, Pendse D, Emberton M (2009) Photodynamic therapy for prostate cancer?a review of current status and future promise. Nat Clin Pract Urol 6(1): 18-30.

40. Lucky SS, Soo KC, Zhang Y (2015) Nanoparticles in photodynamic therapy. Chem Rev 115(4): 1990-2042.

41. Castano AP, Mroz P, Hamblin MR (2006) Photodynamic therapy and antitumour immunity. Nat Rev Cancer 6(7): 535-545.

42. Ge JC, Lan MH, Zhou BJ, Liu WM, Guo L, et al. (2014) A graphene quantum dot photodynamic therapy agent with high singlet oxygen generation. Nat Commun 5: 4596

43. Macdonald IJ, Dougherty TJ (2001) Basic principles of photodynamic therapy. J Porphyrins Phthalocyanines 5(2): 105-129.

44. Guan KW, Jiang YQ Sun CS, Yu H (2011) A two-layer model of laser interaction with skin A photothermal effect analysis. Optics and Laser Technology 43(3): 425-429.

45. Brown S (2008) Photodynamic Therapy - Two photons are better than one. Nat Photonics 2: 394-395. 
46. Mitsunaga M, Ogawa M, Kosaka N, Rosenblum LT, Choyke PL, et al. (2011) Cancer cell-selective in vivo near infrared photoimmunotherapy targeting specific membrane molecules. Nat Med 17(12): 1685-1691.

47. Chen GY, Qiu HL, Prasad PN, Chen XY (2014) Upconversion Nanoparticles: Design, Nanochemistry, and Applications in Theranostics. Chem Rev 114(10): 5161-5214.

48. Liang LE, Care A, Zhang R, Lu YQ, Packer NH, et al. (2016) Facile Assembly of Functional Upconversion Nanoparticles for Targeted Cancer Imaging and Photodynamic Therapy. ACS Appl Mater Interfaces 8(19): 1194511953.

49. Kamkaew A, Cheng L, Goel S, Valdovinos HF, Barnhart TE, et al. (2016) Cerenkov Radiation Induced Photodynamic Therapy Using Chlorin e6-Loaded Hollow Mesoporous Silica Nanoparticles. ACS Appl Mater Interfaces 8(40): 26630-26637.

50. Tang YG, HuJ, Elmenoufy AH, Yang XL (2015) Highly Efficient FRET System Capable of Deep Photodynamic Therapy Established on X-ray Excited Mesoporous LaF3: Tb Scintillating Nanoparticles. ACS Appl Mater Interfaces 7(22): 12261-12269.

51. Chen W, Zhang J (2006) Using nanoparticles to enable simultaneous radiation and photodynamic therapies for cancer treatment. J Nanosci Nanotechnol 6(4): 1159-1166.

52. Hu J, Tang YA, Elmenoufy AH, Xu HB, Cheng Z, et al. (2015) NanocompositeBased Photodynamic Therapy Strategies for Deep Tumor Treatment Small 11(44): 5860-5887.

53. Elmenoufy AH, Tang YA, Hu J, Xu HB, Yang XL (2015) A novel deep photodynamic therapy modality combined with CT imaging established via X-ray stimulated silica-modified lanthanide scintillating nanoparticles. Chemical Communications 51: 12247-12250.

54. Choi M, Choi J, Kim S, Nizamoglu S, Hahn SK, et al. (2013) Light-guiding hydrogels for cell-based sensing and optogenetic synthesis in vivo. Nat Photonics 7: 987-994.

55. Kim TI, McCall JG, Jung YH, Huang X, Siuda ER, et al. (2013) Injectable, Cellular-Scale Optoelectronics with Applications for Wireless Optogenetics. Science 340(6129): 211-216.

56. Bagley AF, Hill S, Rogers GS, Bhatia SN (2013) Plasmonic Photothermal Heating of Intraperitoneal Tumors through the Use of an Implanted Near-Infrared Source. ACS Nano 7(9): 8089-8097.

57. Widder EA (2010) Bioluminescence in the ocean: origins of biological, chemical, and ecological diversity. Science 328(5979): 704-708

58. Herring P J (1977) Bioluminescence of marine organisms. Nature 267: 788-793.

59. Li J, Chen L, Du L, Li M (2013) Cage the firefly luciferin! -a strategy for developing bioluminescent probes. Chem Soc Rev 42(2): 662-676.

60. Ando Y (2007) Development of a quantitative bio/chemiluminescence spectrometer determining quantum yields: Reexamination of the aqueous luminol chemiluminescence standard. Photochem Photobiol 83(5): 1205-1210.

61. El Dakdouki, MH, Xia JG, Zhu DC, Kavunja H, et al. (2014) Assessing the in Vivo Efficacy of Doxorubicin Loaded Hyaluronan Nanoparticles. ACS Appl Mater Interfaces 6(1): 697-705.

62. Yuan H, Chong H, Wang B, Zhu C, Liu L, et al. (2012) Chemical moleculeinduced light-activated system for anticancer and antifungal activities. J Am Chem Soc 134(32): 13184-13187.

63. Xiong L, Shuhendler A J, Rao J (2012) Self-luminescing BRETFRET nearinfrared dots for in vivo lymph-node mapping and tumour imaging. Nat Commun 3: 1193.

64. Hsu CY, Chen CW, Yu HP, Lin YF, Lai PS (2013) Bioluminescence resonance energy transfer using luciferase-immobilized quantum dots for selfilluminated photodynamic therapy. Biomaterials 34(4): 1204-1212.
65. Kim YR, Kim S, Choi JW, Choi SY, Lee SH, et al. (2015) BioluminescenceActivated Deep- Tissue Photodynamic Therapy of Cancer. Theranostics 5(8): 805-817.

66. Cui Z, Nair LS (2010) Chitosan: A Versatile Biomedical Polymer. Recent Patents on Biomedical Engineering 3(3): 129-137.

67. Yang J, Han S, Zheng H, Dong H, Liu J (2015) Preparation and application of micro/nanoparticles based on natural polysaccharides. Carbohydrate Polymers 123: 53-66

68. Pillai CKS, Paul W, Sharma CP (2009) Chitin and chitosan polymers: Chemistry, solubility and fiber formation. Progress in Polymer Science 34(7): 641-678

69. Tiyaboonchai W, Limpeanchob N (2007) Formulation and characterization of amphotericin B-chitosan-dextran sulfate nanoparticles. Int J Pharm 329(1-2): 142-149.

70. Sonaje K, Chen YJ, Chen HL, Wey SP, Juang JH, et al. (2010) Entericcoated capsules filled with freeze-dried chitosan/poly ( $\gamma$-glutamic acid) nanoparticles for oral insulin delivery. Biomaterials 31(12): 3384-3394.

71. Luo Y, Teng Z, Li Y, Wang Q (2015) Solid lipid nanoparticles for oral drug delivery: Chitosan coating improves stability, controlled delivery, mucoadhesion and cellular uptake. Carbohydr Polym 122: 221-229.

72. Duttagupta DS, Jadhav VM, Kadam VJ (2015) Chitosan: A propitious biopolymer for drug delivery. Curr Drug Deliv 12(4): 369-381.

73. Rodrigues S, Costa AMR Da, Grenha A (2012) Chitosan/carrageenan nanoparticles: Effect of cross-linking with tripolyphosphate and charge ratios. Carbohydr Polym 89(1): 282-289.

74. Chen Y, Mohanraj VJ, Parkin JE (2003) Chitosan-dextran sulfate nanoparticles for delivery of an anti-angiogenesis peptide. Letters in Peptide Science 10(5-6): 621-629.

75. Rinaudo M, Pavlov G, Desbrières J (1999) Influence of acetic acid concentration on the solubilization of chitosan. Polymer 40(25): 7029 7032.

76. Saboktakin MR, Tabatabaie RM, Maharramov A, Ramazanov MA (2011) Synthesis and characterization of $\mathrm{pH}$-dependent glycol chitosan and dextran sulfate nanoparticles for effective brain cancer treatment. Int J Biol Macromol 49(4): 747-751.

77. Vårum KM, Myhr MM, Hjerde RJN, Smidsrød O (1997) In vitro degradation rates of partially $\mathrm{N}$-acetylated chitosans in human serum. Carbohydr Res 299(1-2): 99-101.

78. Dhawan S, Singla AK, Sinha VR (2004) Evaluation of mucoadhesive properties of chitosan microspheres prepared by different methods. AAPS PharmSciTech 5(4): e67.

79. Janes KA, Fresneau MP, Marazuela A, Fabra A, Alonso MJ (2001) Chitosan nanoparticles as delivery systems for doxorubicin. J Control Release 73(2-3): 255-267

80. Xiao B, Laroui H, Viennois E, Ayyadurai S, Charania MA, et al. (2014) Nanoparticles with surface antibody against CD98 and carrying CD98 small interfering RNA reduce colitis in mice. Gastroenterology 146(5): 1289-1300.

81. Tekie FSM, Atyabi F, Soleimani M, Arefian E, Atashi A, et al. (2015) Chitosan polyplex nanoparticle vector for miR-145 expression in MCF-7: Optimization by design of experiment. Int J Biol Macromol 81: 828-837.

82. European Food Safety Authority (2011) Scientific opinion on the substantiation of health claims related to chitosan. EFSA J 9(1924): 1-21.

83. Shields KM, Smock N, McQueen CE, Bryant PJ (2003) Chitosan for weight loss and cholesterol management. Am J Heal Syst Pharm 60(13): 13101313.

84. Patti AM, Katsiki N, Nikolic D, Al Rasadi K, Rizzo M (2015) Nutraceuticals in lipid-lowering treatment: A narrative review on the role of chitosan. Angiology 66(5): 416-421. 
85. Huang SS, Sung SH, Chiang CE (2007) Chitosan potentiation of warfarin effect. AnnPharmacother 41(11): 1912-1914.

86. Striano P, Zara F, Minetti C, Striano S (2009) Chitosan may decrease serum valproate and increase the risk of seizure reappearance. BMJ 339: b3751.

87. Cheow WS, Kiew TY, Hadinoto K (2015) Amorphous nanodrugs prepared by complexation with polysaccharides: Carrageenan versus dextran sulfate. Carbohydr Polym 117: 549-558.

88. Hiebert LM, Wice SM, Jaques LB, Williams KE, John M (1999) Orally administered dextran sulfate is absorbed in HIV-positive individuals. J Lab Clin Med 133(2): 161-170.

89. McCarthy RE, Arnold LW, Babcock GF (1977) Dextran sulphate: an adjuvant for cell-mediated immune responses. Immunology 32(6): 963974.

90. Tiyaboonchai W, Woiszwillo J, Sims RC, Middaugh CR (2003) Insulin containing polyethylenimine-dextran sulfate nanoparticles. Int J Pharm 255(1-2): 139-151.

91. Smith KM, Chang RS, Tabba HD, Yongsheng He (1988) Dextran sulfate as an inhibitor against the human immunodeficiency virus (42811). Proc Soc Exp Biol Med 189(3): 304-309.

92. Baba M, Pauwels R, Balzarini J, Arnout J, Desmyter J, et al. (1988) Mechanism of inhibitory effect of dextran sulfate and heparin on replication of human immunodeficiency virus in vitro. Proc Natl Acad Sci U S A 85(16): 6132-6136

93. Chao Y, Makale M, Karmali PP, Sharikov Y, Tsigelny I, et al. (2012) Recognition of dextran-superparamagnetic iron oxide nanoparticle conjugates (feridex) via macrophage scavenger receptor charged domains. Bioconjug Chem 23(5): 1003-1009.

94. Persson UC, Hammarström LTG, Smith CIE (1977) Macrophages are required for the dextran sulfate induced activation of B lymphocytes. Immunol 119(3): 1138-1144.

95. Suzuki K, Nishioka J, Hashimoto S (1979) Inhibition of factor VIIIassociated platelet aggregation by heparin and dextran sulfate, and its mechanism. Biochim Biophys Acta 585(3): 416-426

96. MeshkibafS, Martins AJ, Henry GT, Kim SO (2016) Protective role of G-CSF in dextran sulfate sodium-induced acute colitis through generating guthoming macrophages. Cytokine 78: 69-78.

97. Munyaka PM, Rabbi MF, Khafipour E, Ghia J E (2016) Acute dextran sulfate sodium (DSS)-induced colitis promotes gut microbial dysbiosis in mice. J Basic Microbiol 56(9): 986-998.

98. (2011) Low molecular weight dextran sulfate for the prevention of graft rejection during pancreatic islet transplantation. European Medicines Agency.

99. (2011) Low molecular weight dextran sulfate as treatment for mobilisation of progenitor cells prior to stem cell transplantation. European Medicines Agency.

100. Antony V Samrot, Ujjala Burman, Sheryl Ann Philip, Shobana Na, Kumar Chandrasekaran (2018) Synthesis of curcumin loaded polymeric nanoparticles from crab shell derived chitosan for drug delivery. Informatics in Medicine Unlocked 10: 159- 182

101. Hamideh Moshkbar, Nasser Arsalani, Laleh Saleh Ghadimi (2018) Synthesis of Chitosan/Gelatin granule containing amine derivated octa (ammonium chloride) substituted Polyhedral Oligomeric Silsesquioxane and investigating its application as a drug carrier", International Journal of Polymeric Materials and Polymeric Biomaterials.

102. Haliza Katas, Maria Abdul Ghafoor Raja, Kai Leong Lam (2013) Development of Chitosan Nanoparticles as a Stable Drug Delivery System for Protein/siRNA. International Journal of Biomaterials.

103. Ji Woon Suh, Ji Soo Lee, Sanghoon Ko, Hyeon Gyu Lee (2016) Preparation and Characterization of Mucoadhesive Buccal Nanoparticles Using Chitosan and Dextran Sulfate. J Agric Food Chem 64(26): 5384-5388.
104. Yan Chen, Vellore J Mohanraj, John E Parkin (2003) Chitosan-dextran sulfate nanoparticles for delivery of an anti-angiogenesis Peptide. Letters in Peptide Science 10(5-6): 621-629.

105. Fwu Long Mi, Yu Ru Su, Shu Huei Yu, An Chong Chao (2003) Delivery of Indocyanine Green to Cancer Cells by Chitosan-Based Nanocarriers. J Pharm Sci 92: 2090-2097.

106. Pei Ru Wei, Yaswanth Kuthati, Ranjith Kumar Kankala, Chia Hung Lee (2015) Synthesis and Characterization of Chitosan-Coated Near-Infrared (NIR) Layered Double Hydroxide-Indocyanine Green Nanocomposites for Potential Applications in Photodynamic Therapy. Int J Mol Sci 16(9): 20943-20968.

107. Wagner V, Dullaart A, Bock A, Zweck A (2006) The emerging nanomedicine landscape. Nat Biotechnol 24(10): 1211-1217.

108. Kim BY, Rutka JT, Chan WC (2010) Nanomedicine. N Eng J Med 363(25): 2434-2443.

109. Jacobson GB, Gonzalez Gonzalez E, Spitler R, Shinde R, Leake D et al. (2010) Biodegradable nanoparticles with sustained release of functional siRNA in skin. J Pharm Sci 99(10): 4261-4266.

110. O’Brien ME, Wigler N, Inbar M, Rosso R, Grischke E (2004) Reduced cardiotoxicity and comparable efficacy in a Phase III trial of pegylated liposomal doxorubicin $\mathrm{HCl}$ (CAELYX/doxil) versus conventional doxorubicin for first-line treatment of metastatic breast cancer. Ann Oncol 15(3): 440-449.

111. Davis ME, Zuckerman JE, Choi CH, Seligson D, Tolcher A (2010) Evidence of RNAi in humans from systemically administered siRNA via targeted nanoparticles. Nature 464(7291): 1067-1070.

112. Scranton R, Cincotta A (2010) Bromocriptine - unique formulation of a dopamine agonist for the treatment of Type 2 diabetes. Expert Opin Pharmacother 11(2): 269-279.

113. Lim WT, Tan EH, Toh CK, Hee SW, Leong SS, et al.(2009) Phase I pharmacokinetic study of a weekly liposomal paclitaxel formulation (Genexol-PM) in patients with solid tumors. Ann Oncol 21(2): 382388.

114. Winter PM, Morawski AM, Caruthers SD, Fuhrhop RW, Zhang $\mathrm{H}$, et al. (2003) Molecular imaging of angiogenesis in early-stage atherosclerosis with avb3-integrin-targeted nanoparticles. Circulation 108(18): 2270-2274.

115. Keren S, Zavaleta C, Cheng Z, de la Zerda A, Gheysens O, et al. (2008) Noninvasive molecular imaging of small living subjects using Raman spectroscopy. Proc Natl Acad Sci U S A 105(15): 5844-5849.

116. Jain PK, Huang X, El Sayed IH, El Sayed MA (2008) Noble metals on the nanoscale: optical and photothermal properties and some applications in imaging, sensing, biology, and medicine. Acc Chem Res 41(12): 1578-1586.

117. Maeda H, Wu J, Sawa T, Matsumura Y, Hori K (2000) Tumor vascular permeability and the EPR effect in macromolecular therapeutics: a review. J Control Release 65(1-2): 271-284.

118. Knop K, Hoogenboom R, Fischer D, Schubert US (2006) Poly (ethylene glycol) in drug delivery: pros and cons as well as potential alternatives. Angew Chem. Int Ed Engl 49(36): 6288-6308.

119. van Vlerken LE, Vyas TK, Amiji MM (2007) Poly (ethylene glycol)modified nanocarriers for tumor-targeted and intracellular delivery. Pharm Res 24(8): 1405-1414

120. Kanaras AG, Kamounah FS, Schaumburg K, Kiely CJ, Brust M (2002) Thioalkylated tetraethylene glycol: a new ligand for water soluble monolayer protected gold clusters. Chemical Communications 20: 2294-2295.

121. Kwon GS (2003) Polymeric micelles for delivery of poorly watersoluble compounds. Crit Rev Ther Drug Carrier Syst 20(5): 357-403.

122. Gref R, Minamitake Y, Peracchia MT, Trubetskoy V, Torchilin V, et al. (1994) Biodegradable long-circulating polymeric nanospheres. Science 263(5153): 1600-1603. 
123. Roberts MJ, Bentley MD, Harris JM (2002) Chemistry for peptide and protein PEGylation. Adv Drug Deliv Rev 54(4): 459-476.

124. Li D, He Q, Li J (2009) Smart core/shell nanocomposites: intelligent polymers modified gold nanoparticles. Adv Colloid Interface Sci 149(1-2): 28-38.

125. Prencipe G, Tabakman SM, Welsher K, Goodwin AP, Zhang L, et al. (2009) PEG branched polymer for functionalization of nanomaterials with ultralong blood circulation. J Am Chem Soc 131(13): 4783-4787.

126. Saba TM (1970) Physiology and physiopathology of the reticuloendothelial system. Arch Intern Med 126(6): 1031-1052.

127. Peracchia MT, Fattal E, Desmaële D, Besnard M, Noël JP, et al. (1999) Stealth PEGylated polycyanoacrylate nanoparticles for intravenous administration and splenic targeting. J Control Release 60(1): 121-128.

128. Owens D 3rd, Peppas N (2006) Opsonization, biodistribution, and pharmacokinetics of polymeric nanoparticles. Int J Pharm 307(1): 93102.

129. Decker K (1990) Biologically active products of stimulated liver macrophages (Kupffer cells). Eur J Biochem 192(2): 245-261.

130. Zolnik BS, Sadrieh N (2009) Regulatory perspective on the importance of ADME assessment of nanoscale material containing drugs. Adv Drug Deliv Rev 61(6): 422-427.

131. Guzman K, Finnegan M, Banfield J (2006) Influence of surface potential on aggregation and transport of titania nanoparticles. Environ. Sci Technol 40(24): 7688-7693.

132. Yang P, Ando M, Murase N (2010) Various Au nanoparticle organizations fabricated through $\mathrm{SiO} 2$ monomer induced self-assembly. Langmuir 27(3): 895-901.

133. Jun Y, Casula M, Sim J, Kim SY, Cheon J, et al. (2003) Surfactant-assisted elimination of a high energy facet as a means of controlling the shapes of TiO2 nanocrystals. J Am Chem Soc 125(51): 15981-15985.

134. Förster S, Antonietti M (1998) Amphiphilic block copolymers in structure-controlled nanomaterial hybrids. Advanced Materials 10(3): 195-217.

135. Zhao W, Brook M, Li Y (2008) Design of gold nanoparticle based colorimetric biosensing assays. Chembiochem 9(15): 2363-2371.

136. Sze A, Erickson D, Ren L, Li D (2003) Zeta potential measurement using the Smoluchowski equation and the slope of the current-time relationship in electroosmotic flow. J Colloid Interface Sci 261(2): 402410.

137. Alexis F, Pridgen E, Molnar LK, Farokhzad OC (2008) Factors affecting the clearance and biodistribution of polymeric nanoparticles. Mol Pharm 5(4): 505-515.

138. Strickley RG (2004) Solubilizing excipients in oral and injectable formulations. Pharm Res 21(2): 201-230.

139. Tan JS, Butterfield DE, Voycheck CL, Caldwell KD, Li JT (1993) Surface modification of nanoparticles by PEO/PPO block copolymers to minimize interactions with blood components and prolong blood circulation in rats. Biomaterials 14(11): 823-833.

140. Mueller BG, Kissel T (1993) Camouflage nanospheres: a new approach to bypassing phagocytic blood clearance by surface modified particulate carriers. Pharmaceut Pharmacol Lett 3(2): 67-70.

141. Klibanov AL, Maruyama K, Torchilin VP, Huang L (1990) Amphipathic polyethyleneglycols effectively prolong the circulation time of liposomes. FEBS Lett 268(1): 235-237.

142. Kataoka K, Harada A, Nagasaki Y (2001) Block copolymer micelles for drug delivery: design, characterization and biological significance. Adv Drug Deliv Rev 47(1): 113-131.

143. Abuchowski A, McCoy JR, Palczuk NC, Vanes T, Davis FF (1977) Effect of covalent attachment of polyethylene-glycol on immunogenicity and circulating life of bovine liver catalase. J Biol Chem 252(11): 35823586 .
144. Abuchowski A, Vanes T, Palczuk NC, Davis FF (1977) Alteration of immunological properties of bovine serum-albumin by covalent attachment of polyethylene-glycol. J Biol Chem 252(11): 3578-3581.

145. Arturson P, Laakso T, Edman P (1983) Acrylic microspheres in vivo: Blood elimination kinetics and organ distribution of microparticles with different surface characteristics. J Pharm Sci 72(12): 1415-1420.

146. Petros RA, DeSimone JM (1999) Strategies in the design of nanoparticles for therapeutic applications. Nat Rev Drug Discov 9(8): 615-627.

147. Laginha KM, Verwoert S, Charrois GJ, Allen TM (2005) Determination of doxorubicin levels in whole tumor and tumor nuclei in murine breast cancer tumors. Clin Cancer Res 11(19): 6944-6949.

148. Ahmed M, Lukyanov AN, Torchilin V, Tournier H, Schneider AN, et al. (2005) Combined radiofrequency ablation and adjuvant liposomal chemotherapy: effect of chemotherapeutic agent, nanoparticle size, and circulation time. J Vasc Interv Radiol 16(10): 1365-1371.

149. Gabizon A, Shmeeda H, Barenholz Y (2003) Pharmacokinetics of PEGylated liposomal Doxorubicin: review of animal and human studies. Clin Pharmacokinet 42(5): 419-436.

150. Moreno Aspitia A, Perez EA (2005) Nanoparticle albumin-bound paclitaxel (ABI-007): a newer taxane alternative in breast cancer. Future Oncol 1(6): 755-762.

151. Thadakapally R, Aafreen A, Aukunuru J, Habibuddin M, Jogala S (2016) Preparation and Characterization of PEG-albumin-curcumin Nanoparticles Intended to Treat Breast Cancer. Indian J Pharm Sci 78(1): 65-72.

152. Gao S, Wang G, Qin Z, Wang X, Zhao G, et al. (2017) Oxygen-generating hybrid nanoparticles to enhance fluorescent/photoacoustic/ ultrasound imaging guided tumor photodynamic therapy. Biomaterials 112: $324-335$

153. Luo T, Zhang Q, Lu QB (2017) Combination of near infrared lightactivated photodynamic therapy mediated by indocyanine green with etoposide to treat non-small-cell lung cancer. Cancers(Basel) 9(6): E63.

154. Zhang S, Guo W, Wei J, Li C, Liang X J, et al. (2017) Terrylenediimidebased intrinsic theranostic nanomedicines with high photothermal conversion efficiency for photoacoustic imaging-guided cancer therapy. ACS Nano 11: 3797-3805.

155. Dong Z, Gong H, Gao M, Zhu W, Sun X, et al. (2016) Polydopamine nanoparticles as a versatile molecular loading platform to enable imaging-guided cancer combination therapy. Theranostics 6(7): 10311042.

156. Song S, He S, Tao Y, Wang L, Han F, et al. (2017) Indocyanine green loaded magnetic carbon nanoparticles for near infrared fluorescence/ magnetic resonance dual-modal imaging and photothermal therapy of tumor. ACS Appl Mater Interfaces 9(11): 9484-9495.

157. Li X, Xing L, Hu Y, Xiong Z, Wang R, et al. (2017) An RGD-modified hollow silica@Au core/shell nanoplatform for tumor combination therapy. Acta Biomaterialia 62: 273-283.

158. Chu CK, Tu YC, Hsiao JH, Yu JH, Yu CK, et al. (2016) Combination of photothermal and photodynamic inactivation of cancer cells through surface plasmon resonance of a gold nanoring. Nanotechnology 27(11): 115102.

159. Jia Q, Ge J, Liu W, Liu S, Niu G, et al. (2016) Gold nanorod@silicacarbon dots as multifunctional phototheranostics for fluorescence and photoacoustic imaging-guided synergistic photodynamic/ photothermal therapy. Nanoscale 8: 13067-13077.

160. Peng S, He Y, Er M, Sheng Y, Gu Y, et al. (2017) Biocompatible CuS-based nanoplatforms for efficient photothermal therapy and chemotherapy in vivo. Biomater Sci 5(3): 475-484.

161. Wang W, Wang L, Liu S, Xie Z (2018) Metal-organic frameworks@ polymer composites containing cyanines for near-infrared fluorescence imaging and photothermal tumor therapy. Bioconjugate Chem 28: 2784-2793. 
162. Poland CA, Duffin R, Kinloch I, Maynard A, Wallace WAH, et al. (2008) Carbon nanotubes introduced into the abdominal cavity of mice show asbestos-like pathogenicity in a pilot study. Nat Nanotechnol 3(7): 423-428.

163. Liu H, Liu T, Wu X, Li L, Tan L, et al. (2012) Targeting gold nanoshells on silica nanorattles: A drug cocktail to fight breast tumors via a single irradiation with near-infrared laser light. Adv Mater 24: 755-761.

164. Li X, Xing L, Zheng K, Wei P, Du L, et al. (2017) Formation of gold nanostar-coated hollow mesoporous silica for tumor multimodality imaging and photothermal therapy. ACS Appl Mater Interfaces 9(7): 5817-5827.

165. Liu Z, Fan AC, Rakhra K, Sherlock S, Goodwin A, et al. (2009) Supramolecular stacking of doxorubicin on carbon nanotubes for in vivo cancer therapy. Angew Chem Int Ed 48(41): 7668-7672.
166. Jian WH, Yu TW, Chen CJ, Huang WC, Chiu HC, et al. (2015) Indocyanine green-encapsulated hybrid polymeric nanomicelles for photothermal cancer therapy. Langmuir 31(22): 6202-6210.

167. Wu M, Wang Q Zhang D, Liao N, Wu L, et al. (2016) Magnetite nanocluster@poly(dopamine)-PEG@ indocyanine green nanobead with magnetic field-targeting enhanced MR imaging and photothermal therapy in vivo. Colloids Surf B 141: 467-475.

168. Guan S, Weng Y, Li M, Liang R, Sun C, et al. (2017) An NIR-sensitive layered supramolecular nanovehicle for combined dual-modal imaging and synergistic therapy. Nanoscale 9: 10367-10374.

169. Liu Y, Zhi X, Yang M, Zhang J, Lin L, et al. (2017) Tumor-triggered drug release from calcium carbonate-encapsulated gold nanostars for near-infrared photodynamic/photothermal combination antitumor therapy. Theranostics 7(6): 1650-1662. 